\title{
Diagnóstico del proceso de inspección mediante índices de capacidad
}

\author{
Diagnosis of the inspection process through capability indices \\ José Daniel Mosquera-Artamonov ${ }^{1} \quad$ Irina Artamonova $^{2} \quad$ Julio César Mosquera $^{3}$ \\ Recibido 25 de julio de 2012, aceptado 12 de agosto de 2013 \\ Received: July 25, 2012 Accepted: August 12, 2013
}

\begin{abstract}
RESUMEN
Este trabajo presenta una metodología de evaluación de la capacidad de un proceso usando como herramienta estadística los denominados Índices de Capacidad. El problema se enfoca en el proceso de inspección de una empresa mexicana de medicamentos para animales, en la cual se realizó un análisis de la capacidad de los proveedores en cumplir con las especificaciones del departamento de calidad y la capacidad que tiene el departamento de inspección en la detección de productos defectuosos. Los datos analizados corresponden a inconformidades presentadas en el segundo periodo del 2011. Se detectó que los diferentes proveedores existentes en la empresa no tienen capacidad para cumplir con los límites de especificación de la empresa y por último el proceso de inspección no tiene capacidad suficiente en la detección de productos defectuosos por lo cual se recomienda realizar un ajuste en el plan de muestreo de la empresa.
\end{abstract}

Palabras clave: Índices de capacidad, inspección, prueba de bondad del ajuste, cartas de control, transformación Johnson.

\begin{abstract}
This work presents an evaluation methodology on the capacity a particular process using a set of statistical tools known as Capacity Indexes. The main problem focuses on the inspection process in a Mexican pharmaceutical company specialized on animals, where analysis were performed concerning the capacities of different providers to meet the required specifications, designated by the company's quality department and also on the response time on their own inspection department to test their capacity to detect defective supplies. The data analyzed corresponds to unconformities presented during the second period of 2011. It was detected that the current suppliers do not have the capacity to comply with the required quality specifications and finally, the inspection process didn't have the capacity to detect defective products, whereby it is recommended to perform adjustments on their current sampling procedures of the pharmaceutical company.
\end{abstract}

Keywords: Capability indices, inspection, testing goodness of fit, control charts, Johnson transform.

\section{INTRODUCCIÓN}

Con el paso de los años la calidad de la inspección de la materia prima y del producto terminado ha tenido gran importancia para la satisfacción del cliente final del producto [1]. Esa necesidad de manufacturar productos con niveles de calidad competitivos ha generado diferentes métodos de inspección [2-4]. Esa necesidad de satisfacer a clientes cada vez más exigentes se ve reflejada en la gran importancia

1 Facultad de Ingeniería. División de Estudios de Posgrado. Universidad Autónoma de Querétaro. Querétaro, México. E-mail: Xoce15@ingenieros.com

2 Facultad de Física. Universidad del Quindío. Armenia. Colombia. E-mail: artiri@ gmail.com

3 Instituto Interdisciplinario de las Ciencias. Facultad de Física. Universidad del Quindío. Armenia. Colombia.

E-mail: jcmosquera@uniquindio.edu.co 
dada en la actualidad a los procesos de control de calidad tanto desde un enfoque de producción como de normatividad [5].

Bajo esta óptica las empresas se certifican en diferentes normas (ISO 9001, 14000, 9004, entre otras). Al momento de encontrar una inconformidad en los procesos productivos de la empresa se enciende una alarma que en muchos casos tiene como consecuencia el despido del personal responsable, dadas las repercusiones económicas [6], y de desprestigio que se genera al enviar un producto no conforme al cliente [7]. Por este motivo es tan importante en las empresas del siglo XXI tener procesos de inspección controlados en los cuales se verifique la calidad de los insumos o productos que entran a la empresa como los que salen de la misma.

Una de las estrategias usadas para la mejora de procesos tanto en empresas de manufactura [8] como de servicios [9], hospitales [10] entre otras, es la metodología DMAIC (Definir, Medir, Analizar, Mejorar, Controlar), la cual busca tener cambios mesurables y trascendentales para los procesos que se están analizando. Esta metodología es un proceso iterativo que se rige por una estructura enfocada en el trazado de una hipótesis [11].

Dicha metodología está fundamentada en la filosofía de Seis Sigma en donde se busca tener 3,4 partes por millón de defectos en la organización.

El fundamento central de la metodología es la correcta definición del problema a solucionar, dado que todo el esfuerzo del equipo investigador está centrado en dicha meta. Sin embargo primeramente se hace necesario contar con una metodología eficaz de medición de la capacidad del proceso. Por lo cual, el objetivo central de este trabajo es la presentación del uso de los Índices de Capacidad de Proceso aplicados a un caso particular.

\section{PLANTEAMIENTO DEL PROBLEMA}

El presente trabajo se desarrolla en una empresa dedicada a la producción de medicamentos para animales ubicada en la ciudad de Santiago de Querétaro, México, con 42 años de operación. Dicha empresa ha presentado problemas con los proveedores de cajas de almacenamiento de los medicamentos elaborados. Puesto que un proveedor envió en un mismo lote cajas con diferentes especificaciones técnicas (2011, II semestre), se alertó a la dirección sobre el trabajo realizado por el proceso de inspección y la validez del sistema de trabajo.

Por órdenes del departamento de calidad se usa un muestreo basado en la militar estándar con nivel general de inspección II, teniendo un tamaño del lote de 1201 a 3200 unidades [12].

Desde ese momento el equipo de inspección empezó a muestrear el doble de unidades requeridas para tener confianza en el proceso y poder detectar los productos defectuosos del proveedor, recurriendo a un aumento en horas hombre para realizar este proceso, afectando directamente la disponibilidad de los trabajadores del área en la realización de otras labores. Esto incrementó los costos operativos y disminuyó la productividad de la empresa.

La coordinadora de calidad requirió determinar la validez de su proceso de muestreo, en caso contrario que no sea válido reestructurarlo. Adicionalmente se pidió determinar la capacidad de los proveedores para responder a las especificaciones de calidad de la empresa puesto que se ha incurrido en aumento del costo de inspección al tener una persona dedicada a realizar estas funciones. No se debe olvidar que realizar dicho reproceso, desde la óptica de manufactura esbelta así como de la financiera, se considera una actividad que no añade valor al producto.

\section{MATERIALES Y MÉTODOS}

Para poder determinar la efectividad del proceso de inspección se solicitó a la coordinadora de calidad los registros históricos del proceso discriminados por proveedor y los registros de los productos rechazados por el proceso de producción como última instancia. Buscando de esta manera poder evaluar a los proveedores y al proceso mismo.

Para el análisis del proceso se usó el índice de capacidad (ICP), dicho término fue originado por primera vez por Juran [14], donde la fórmula utilizada [11] se presenta a continuación:

$$
I C P=C p \frac{L S E-L I E}{6 \hat{\sigma}}
$$


Donde:

LSE: Límite Superior Especificado

LIE: Límite Inferior Especificado

$\hat{\sigma}$ : Desviación estándar de los datos

Según los valores obtenidos del ICP, se puede conocer la habilidad de cumplir con las especificaciones del proceso como muestra la Tabla 1.

Tabla 1. Análisis del ICP.

\begin{tabular}{|c|c|}
\hline ICP & Decisión \\
\hline $\mathrm{ICP}>1,33$ & Más que adecuado. \\
\hline $1<\mathrm{ICP}<1,33$ & $\begin{array}{c}\text { Adecuado para lo que fue } \\
\text { diseñado. }\end{array}$ \\
\hline $0,67<\mathrm{ICP}<1$ & $\begin{array}{c}\text { No es adecuado para cumplir con } \\
\text { el diseño inicial. }\end{array}$ \\
\hline
\end{tabular}

Para determinar los diferentes índices de capacidad de un proceso, este debe cumplir con dos condiciones esenciales:

1. El proceso debe estar bajo control según explicación detallada contenida en el manual de control de proceso.

2. Los datos se ajustan a una distribución normal.

Cuando los datos no se ajustan a una distribución normal, se debe realizar una estimación de la distribución o transformación escogiendo la que presente mejor ajuste a los datos recolectados del proceso evaluado [15-16].

Cabe la pena aclarar que existen muchos otros indicadores de capacidad entre los que están: Cpm [17], Cpmk [18], PCI [19], Cpp [20], Cs [21], Cpw [22], Spmk [23], CCpk [24], Cpcu [25] entre muchos otros. Convirtiendo la tarea de determinar la capacidad de un proceso en una labor complicada, debido al abanico de posibilidades en metodologías factibles a aplicar en un proceso.

Por otra parte es necesario resaltar que para un proceso que no siga una distribución normal, no se puede estimar indicadores de capacidad a corto plazo como Cp, Cpk, CPU y CPL (Tabla 2) y solo es posible calcular los índices de capacidad de largo plazo o globales, que son Pp, Ppk, PPU y PPL.

En la Tabla 2 se muestran algunas referencias sobre cuándo usar cada uno de los índices, siendo estos indicadores de capacidad los más difundidos en el ámbito industrial.

Tabla 2. Descripción de los ICP.

\begin{tabular}{|l|l|l|}
\hline \multicolumn{1}{|c|}{ Índice } & \multicolumn{1}{|c|}{ Uso } & \multicolumn{1}{c|}{ Fórmula } \\
\hline Cp o Pp & $\begin{array}{l}\text { El proceso está centrado en } \\
\text { los límites de especificación }\end{array}$ & $(\mathrm{LSE}-\mathrm{LIE}) / 6 \sigma$ \\
\hline Cpk o Ppk & $\begin{array}{l}\text { El proceso no está } \\
\text { centrado en los límites de } \\
\text { especificación }\end{array}$ & $\begin{array}{l}\mathrm{Min}\{(\mathrm{LSE}- \\
\mu) / 3 \sigma,(\mu- \\
\mathrm{LIE}) / 3 \sigma\}\end{array}$ \\
\hline $\begin{array}{l}\text { CPU o } \\
\text { PPU }\end{array}$ & $\begin{array}{l}\text { El proceso solo tiene un } \\
\text { límite de especificación } \\
\text { superior }\end{array}$ & $(\mathrm{LSE}-\mu) / 3 \sigma$ \\
\hline $\begin{array}{l}\text { CPL o } \\
\text { PPL }\end{array}$ & $\begin{array}{l}\text { El proceso solo tiene un } \\
\text { límite de especificación } \\
\text { inferior }\end{array}$ & $(\mu-\mathrm{LIE}) / 3 \sigma$ \\
\hline
\end{tabular}

Lo que se debe considerar es: si los datos tomados del proceso que se está analizando siguen una distribución normal, se puede diseñar los ICP de corto plazo y también los de largo plazo [26]. La capacidad global o a largo plazo dice cómo se está comportando el proceso respecto de las especificaciones prefijadas.

Si se quieren calcular los ICP para el número de productos defectuosos se puede usar la distribución de Poisson. Para la característica cualitativa que el producto posee o no (correcto o defectuoso, por ejemplo), puede usarse la distribución binomial. En el caso de esta investigación se estudia la proporción de defectuosos y no el número mismo de ellos. En este caso el tamaño de la muestra es grande y la proporción de defectuosos no es muy cercana a 0 o a 1 , en consecuencia los ICP se estudian a partir de una distribución binomial cuyos percentiles pueden calcularse usando percentiles de la distribución normal estándar [25].

\section{ANÁLISIS}

Lo primero que se realizó fue el análisis de las proporciones de los productos defectuosos enviados por los diferentes proveedores (Figuras 1-4). En esta etapa no se verificó que el proceso de cada proveedor estuviera bajo control, dado que sobre estos procesos por el momento no se puede tomar correctivos en el proceso de manufactura. Con posterioridad se hizo un análisis de capacidad y por último se evaluó el plan del muestreo. 


\section{Proveedor 1}

El primer proveedor analizado fue Pharmaplast. La Figura 1 muestra la variación de los productos defectuosos para el último semestre.

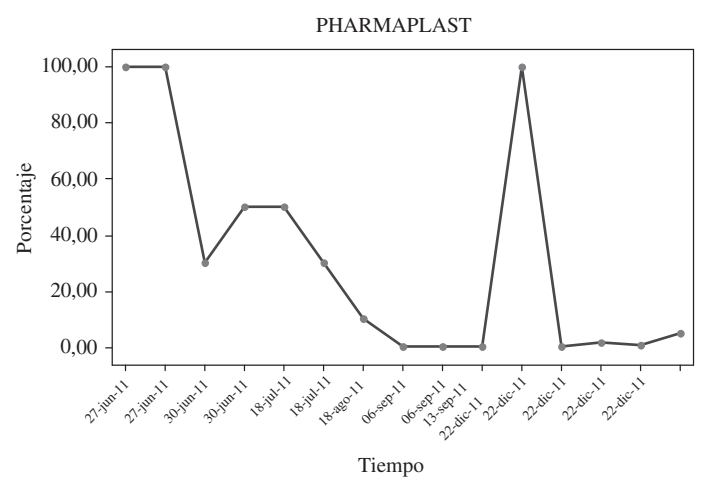

Figura 1. Variación de defectuosos para PHARMAPLAST.

Para poder determinar los ICP se hace una prueba de bondad del ajuste (Tabla 3) con la cual se determinó la función de distribución estadística que presenta mejor ajuste a los datos. Se selecciona la distribución o transformación que presente el valor p (P-Value) mayor a 0,05. En el caso de que existan varias distribuciones que cumplan con este requisito se escoge la que tenga el mayor valor de probabilidad $\mathrm{P}$.

Tabla 3. Prueba de bondad del ajuste para el proveedor 1.

\begin{tabular}{|l|r|}
\hline \multicolumn{1}{|c|}{ Distribución } & \multicolumn{1}{c|}{ P } \\
\hline Normal & $1,386<0,005$ \\
\hline Transformación Box-Cox & 0,020 \\
\hline Lognormal & 0,020 \\
\hline Lognormal de 3 parámetros & $*$ \\
\hline Exponencial & $<0,003$ \\
\hline Exponencial de 2 parámetros & $<0,010$ \\
\hline Weibull & 0,033 \\
\hline Weibull de 3 parámetros & 0,044 \\
\hline Valor extremo más pequeño & $<0,010$ \\
\hline Valor extremo más grande & $<0,010$ \\
\hline Gamma & 0,095 \\
\hline Gamma de 3 parámetros & $*$ \\
\hline Logística & $<0,005$ \\
\hline Loglogística & 0,015 \\
\hline Loglogística de 3 parámetros & $*$ \\
\hline Transformación de Johnson & 0,182 \\
\hline
\end{tabular}

Para poder determinar la ocurrencia de fallas en el proveedor Pharmaplast, las fallas se reagruparon en dos categorías: el estilo del frasco, considerando problemas de etiquetas, color, entre otros, y las dimensiones de los frascos. La primera categoría representó el 99,6\% de los defectos, el restante a $0,04 \%$ está en la segunda categoría.

La prueba de bondad de ajuste indica que la transformación de Johnson (Tabla 3) es la que mejor se ajusta a los datos. Conociendo esto fue realizado el cálculo de los índices de capacidad del proceso (Figura 4), en donde el Ppk es igual a -0,01. Los límites de especificación (LE) transformados para este proceso son: LEI:-0,13621 y LES: -0,00145063.

Con la ayuda del área de calidad de la empresa se establecieron límites para los productos defectuosos (LEI 5\%, LES 9\%) no siendo tan exigentes dado principalmente a que el proceso inspeccionado no tiene tanta significancia en el consumidor final (la mascota). Con estos parámetros se analizó la capacidad que tiene el proveedor para realizar productos sin defectos.

Los LE son mostrados en la Figura 2 siendo las líneas punteadas y en el recuadro izquierdo de la Figura 2 son presentados los valores de los LE transformados con el modelo ajustado.

El Ppk calculado para el proveedor $1(-0,01)$ hace referencia a que el proveedor de insumos no tiene la capacidad para producir material no defectuoso, bajo los límites de especificación mencionados por el departamento de calidad de la empresa.

\section{Proveedor 2}

En la Figura 3 se muestra como la producción del proveedor 2 fue mejorando considerablemente la calidad de las cajas. Es de aclarar que todos los defectos presentados por este proveedor son defectos menores, concernientes a variaciones en las tonalidades en las cajas o manchas en las figuras. De los dos proveedores es el que muestra una mejora continua en su proceso de manufactura de cajas.

A continuación se muestra la prueba de bondad del ajuste para el proveedor 2 (Tabla 4) para determinar cuál modelo estadístico será usado con el fin de conocer la capacidad del proveedor en cumplir con los requisitos mínimos de calidad de la empresa. Para 


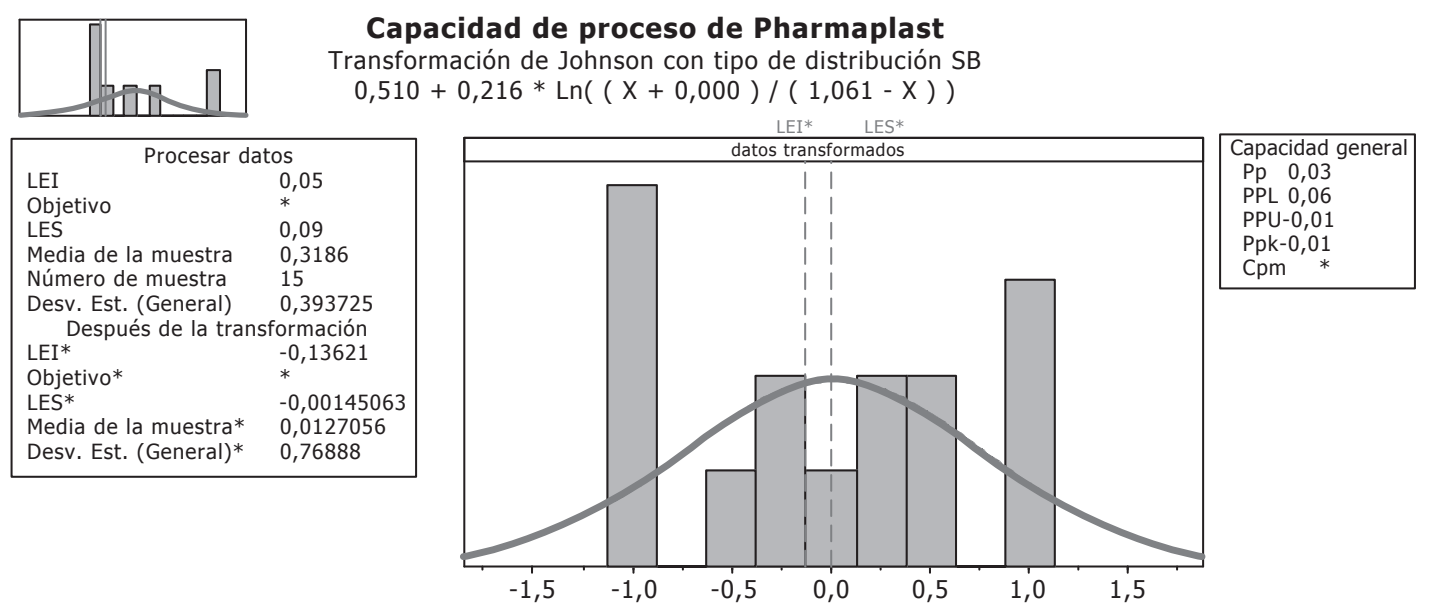

Figura 2. Capacidad de proceso para Pharmaplast.

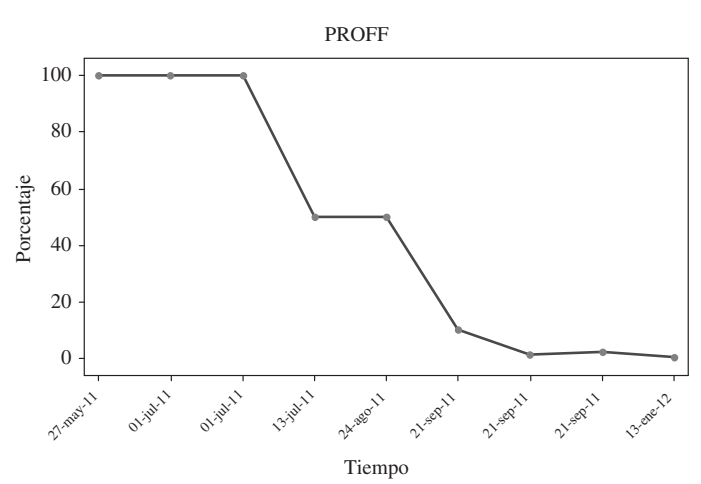

Figura 3. Variación de defectuosos en PROFF.

este proveedor la transformación de Johnson es la que presenta mejor ajuste con un valor $\mathrm{P}$ de 0,271.

Los LE transformados para el proveedor 2 son: LEI -0,49243 y LES -0,320576. Los límites de especificación antes de la transformación son los mismos para los dos proveedores. Después de la transformación de Johnson los LEI y LES se muestran en la Figura 4 con las líneas punteadas rojas, adicionalmente los LE, la media y desviación transformados son presentados en el recuadro de la izquierda.

Aunque se esperaba que la capacidad del proceso para el proveedor 2 fuera la mejor de los proveedores analizados, los estadísticos elaborados por medio de la transformación de Johnson (Ppk $=-0,11)$ muestran que el proceso del proveedor 2 es muy similar al proveedor 1 .
Tabla 4. Prueba de bondad del ajuste para el segundo proveedor

\begin{tabular}{|l|r|}
\hline \multicolumn{1}{|c|}{ Distribución } & \multicolumn{1}{c|}{ P } \\
\hline Normal & 0,040 \\
\hline Transformación Box-Cox & 0,093 \\
\hline Lognormal & 0,079 \\
\hline Lognormal de 3 parámetros & $*$ \\
\hline Exponencial & 0,010 \\
\hline Weibull & 0,064 \\
\hline Weibull de 3 parámetros & 0,05 \\
\hline Valor extremo más pequeño & 0,022 \\
\hline Gamma & 0,139 \\
\hline Gamma de 3 parámetros & \\
\hline Logística & 0,031 \\
\hline Loglogística & 0,061 \\
\hline Loglogística de 3 parámetros & $*$ \\
\hline Transformación de Johnson & 0,271 \\
\hline
\end{tabular}

El departamento de calidad de la empresa tenía la hipótesis que el proveedor con más bajo rendimiento de calidad era el proveedor número 1. Pero bajo los análisis realizados se puede afirmar que no hay una diferencia significativa entre estos dos proveedores apoyado por el valor $\mathrm{p}(\mathrm{P}$-value $=$ $1,000)$, determinado bajo una prueba de proporciones usando el estadístico de Fisher.

\section{Análisis del proceso de inspección}

Para el análisis del proceso de inspección se decidió usar una carta de control, puesto que este proceso sí debe estar bajo control. Escogiendo la carta de control $\mathrm{P}$ que mide los productos defectuosos en 


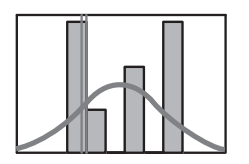

\begin{tabular}{|ll|}
\hline \multicolumn{2}{|c|}{ Procesar datos } \\
LEI & 0,05 \\
Objetivo & $*$ \\
LES & 0,09 \\
Media de la muestra & 0,459389 \\
Número de muestra & 9 \\
Desv. Est. (General) & 0,448778 \\
\multicolumn{2}{|c|}{ Después de la transformación } \\
LEI* & $-0,492436$ \\
Objetivo* & $*$ \\
LES* & $-0,320576$ \\
Media de la muestra* & $-0,0329284$ \\
Desv. Est. (General)* & 0,850998 \\
\hline
\end{tabular}

Transformación de Johnson con tipo de distribución SB

$0,362+0,274 * \operatorname{Ln}((X-0,000) /(1,175-X))$

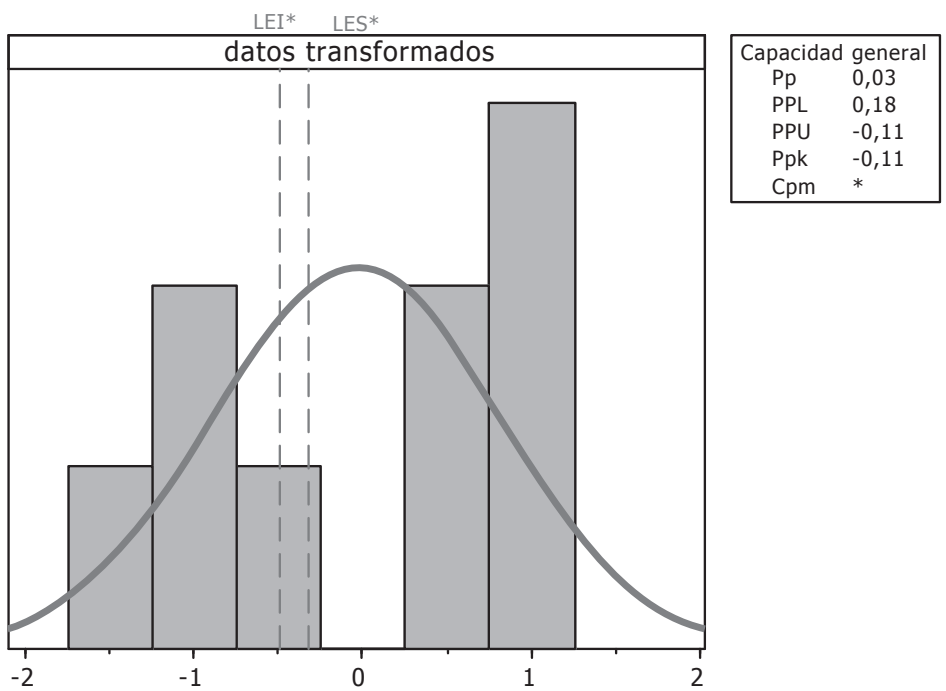

Figura 4. Capacidad del proceso para PROFF.

un lote variable. La Figura 5 muestra unos límites de control superior (LCS) variables, dado que los lotes de producción también son variables. Existiendo cuatro puntos fuera de control, los cuales son evidenciados en los primeros periodos de recolección de la información.

En la Figura 5, el límite inferior en este caso no es de preocupación. Cabe recordar que se analizó la proporción de los productos defectuosos, al tener menos defectos en producción la calidad del producto final es mejor. El proceso de muestreo tuvo un momento fuera de control (los cuatro

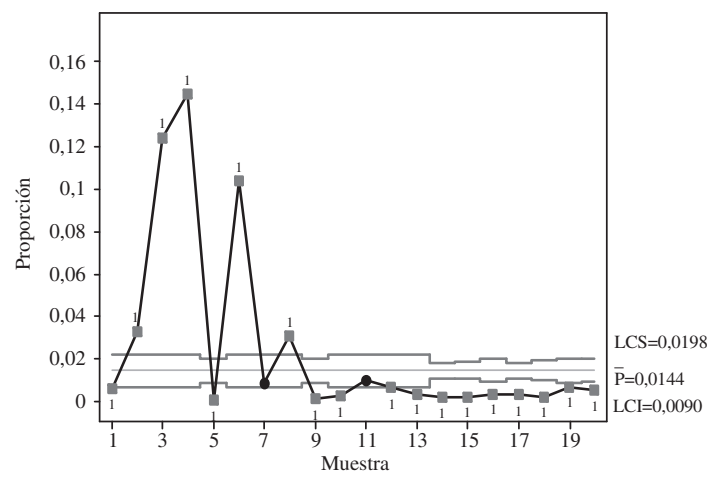

Las pruebas se realizaron con tamaños de la muestra desiguales.

Figura 5. Cartas de control para inspección. puntos superiores), pero después bajo unos ajustes al proceso los niveles de defectuosos no detectados disminuyeron.

Para finalizar se analiza la capacidad del proceso de inspección, usando como modelo de ajuste a los datos la transformación de Johnson (P-value $=0,753$ ). Para el cálculo del Ppk se tuvo en consideración un LSE de 3\%. Es decir, el proceso de inspección no detectará como máximo el $3 \%$ de defectos existentes en las materias primas.

Permitiendo afirmar que el área de inspección no tiene la capacidad (Ppk $=0,32)$ para cumplir con el objetivo propuesto (LSE $=3 \%$ ) (Figura 6). Por consiguiente el plan de muestreo no es el adecuado para cumplir con estas especificaciones en el proceso de inspección. En los tres procesos analizados (dos proveedores e inspección) se usó el indicador Ppk dado que los procesos no estaban centrados en los límites de especificación (Figuras 2, 4 y 6).

\section{CONCLUSIONES}

Para los dos proveedores analizados, a los dos se les pudo realizar el análisis de capacidad dado que presentaban buen ajuste a un modelo estadístico bajo la transformación Johnson. 


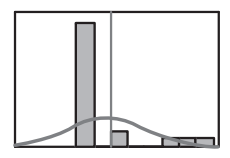

\begin{tabular}{|ll|}
\hline \multicolumn{2}{|c|}{ Procesar datos } \\
LEI & $*$ \\
Objetivo & $*$ \\
LES & 0,03 \\
Media de la muestra & 0,0248443 \\
Número de muestra & 20 \\
Desv.Est. (General) & 0,0442457 \\
Después de la transformación \\
LEI* & $*$ \\
Objetivo* & $*$ \\
LES* & 1,1014 \\
Media de la muestra* & 0,0672716 \\
Desv.Est. (General)* & 1,09377 \\
\hline
\end{tabular}

Trapacidad de proceso para Inspeccio

Transformación de Johnson con tipo de distribución SU $-1,032+0,456 *$ Asenh $((X-0,002) / 0,001)$

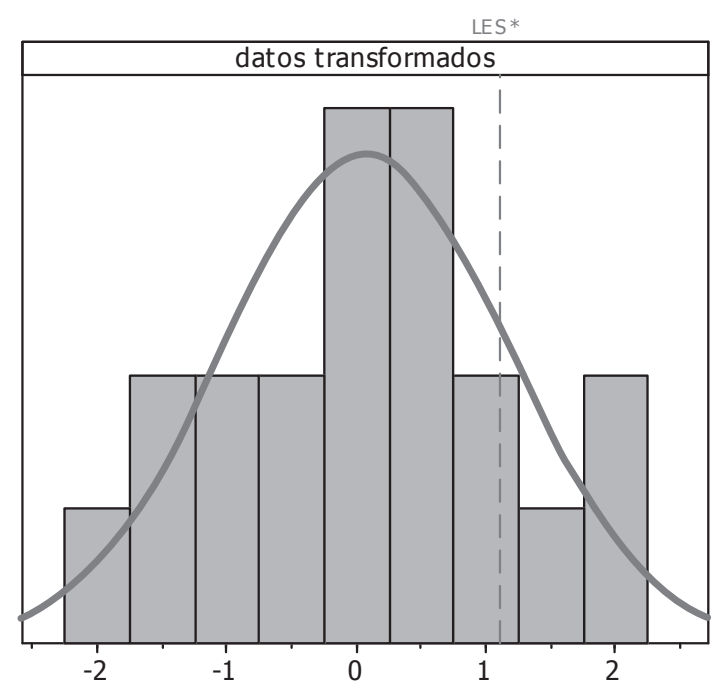

\begin{tabular}{|cc|}
\hline Capacidad general \\
Pp & $*$ \\
PPL & $*$ \\
PPU & 0,32 \\
Ppk & 0,32 \\
Cpm & $*$ \\
\hline
\end{tabular}

Figura 6. Capacidad del proceso de inspección.

Un supuesto de la dirección de calidad de la empresa era que el proveedor 1 tendría menor capacidad de proceso en comparación con el proveedor 2 debido a su historial de incidencia en defectos, pero los proveedores 1 y 2 presentan igual capacidad puesto que los datos históricos no presentan diferencia estadística significativa (Prueba de igualdad de proporciones, $\mathrm{P}$-Value $=1,000)$.

La diferencia real de un proveedor a otro radica principalmente en la ocurrencia de defectos graves en sus producciones, muchos de los defectos registrados son defectuosos permisibles; el proveedor 2 no presenta defectos graves en su historia, pero el proveedor 1 presentó dos veces defectos graves para la coordinación de calidad de la empresa.

En la evaluación del proceso de inspección se determinó que el proceso no tiene capacidad para cumplir con los límites de control propuestos (LSE $=3 \%$ ) por lo cual se recomienda aumentar el nivel de inspección general a III y dentro de tres meses analizar nuevamente los datos recolectados para determinar el efecto del cambio del plan de muestreo.

\section{AGRADECIMIENTOS}

Los autores dan las más sinceras gracias a la empresa, por la facilitación de la información que dio resultado el presente artículo y a la Coordinadora de Calidad, por las aclaraciones realizadas durante el proceso de análisis de la información.

J.D. Mosquera-Artamonov agradece a la Universidad Autónoma de Querétaro por la beca de colegiatura. A la Unidad de Vinculación Tecnológica y Proyectos Especiales de la Universidad Autónoma de Querétaro, por el apoyo en el desarrollo de la presente investigación.

Los autores agradecen a los evaluadores de este trabajo, los que contribuyeron en la refinación de las metodologías e ideas presentadas en el artículo.

\section{REFERENCIAS}

[1] G. Pérez, B. Giraldo y J. Serna. "El mejoramiento de procesos y su aplicación bajo norma ISO 9004: caso compañía de aceites". Dyna. Vol. 73 No 150, pp. 97-106. 2006. ISSN: 0012-7353. Fecha de consulta: 8 de abril de 2013. URL: http://redalyc.uaemex. $\mathrm{mx} / \mathrm{src} / \mathrm{inicio} /$ ArtPdfRed.jsp?iCve=49615014

[2] E. Jaramillo, A. Prieto y P. Boulanger "Inspección de piezas 3D: revisión de la literatura". Ingeniería e Investigación. Vol. $27 \mathrm{~N}^{\circ} 3$, pp. 118-126. 2007. ISSN: 0120-5609. Fecha de consulta: 8 de abril 
de 2013 URL: http://redalyc.uaemex.mx/ redalyc/pdf/643/64327314.pdf

[3] J.A. Cortés Osorio, F.A. Medina Aguirre y J.A. Mendoza Vargas. "Sistema de visión por computador para el control de calidad en la producción". Scientia Et Technica. Vol. XVI No 45, pp. 130-134. 2010. ISSN: 01221701. Fecha de consulta: 8 de abril de 2013 . URL: http://redalyc.uaemex.mx/src/inicio/ ArtPdfRed.jsp?iCve $=84917249023$

[4] D. Mishra and A. Mishra. "Simplified software inspection process in compliance with international standards". Computer Standards \& Interfaces. Vol. 31, Issue 4, pp. 763-771. 2009. ISSN: 0920-5489.

[5] C. Rodríguez, R. Artiles y F. Mujica, "Mejoramiento del proceso de secado de Nepe de una planta”. Revista ingeniería. Vol. $16 \mathrm{~N}^{\circ} 2$, pp. 59-64. 2009. ISSN: 13166832. Fecha de consulta: 8 de abril de 2013 URL: http://redalyc.uaemex.mx/src/inicio/ ArtPdfRed.jsp?iCve=70717501009

[6] A.K. Gramopadhye, B.J. Melloy, M. Gopinath and M. Budgavi. "An evaluation of economic and performance feedback in an inspection task with explicit economic consequences" International Journal of Industrial Ergonomics. Vol. 20, Issue 4, pp. 327-337. 1997. ISSN: 0169-8141.

[7] I. Bendavid, I. and Y.T. Herer. "Economic optimization of off-line inspection in a process that also produces non-conforming units when in control and conforming units when out of control". European Journal of Operational Research. Vol. 195, Issue 1, pp. 139-155. 2009. ISSN: 0377-2217.

[8] R.D. Guevara y J.A. Vargas. "Intervalos de confianza los índices de capacidad Cpm y Cpmk en procesos Gaussianos" Revista Colombiana de Estadística, pp. 153-162. 2006. ISSN: 0120-1751. Fecha de consulta: 8 de abril de 2013 URL: http://www.scielo. org.co/pdf/rce/v29n2/v29n2a02.pdf

[9] T. Jin, B. Janamanchi and Q. Feng. "Reliability deployment in distributed manufacturing chains via closed-loop Six Sigma methodology". International Journal of Production Economics. Vol. 30, Issue 1, pp. 96-103. 2011.

[10] S.V. Goel and V. Chen. "Integrating the global enterprise using Six Sigma: Business process reengineering at General Electric Wind Energy". International Journal of Production Economics. Vol. 113, Issue 2, pp. 914-927. 2008.

[11] C.L. Leaphart, T.A. Gonwa, M.L. Mai, M.B. Prendergast, H.M. Wadei, J.J. Tepas and C.B. Taner. "Formal quality improvement curriculum and DMAIC method results in interdisciplinary collaboration and process improvement in renal transplant patients" Journal of Surgical Research. Vol. 177, Issue 1, pp. 7-13. 2012.

[12] R.G. Schroeder, K. Linderman, C. Liedtke, and A.S. Choo. "Six sigma: definition and underlying theory". Journal of Operations Management. Vol. 26, Issue 4, pp. 536-554. 2008.

[13] Montgomery. "Control estadístico de la calidad. México”. Limusa, S.A. 1999.

[14] J.M. Juran. "Quality Control Handbook". McGraw-Hill. 3 edition. New York. 1974.

[15] M.J.C. Mosquera, A.J.D. Mosquera y V.P.D. Medina. "Evaluación del índice de capacidad del proyecto de deserción académica en la universidad tecnológica de Pereira (UTP)". Revista en Educación en Ingeniería. Vol. 9, pp. 96-103. 2010. Fecha de consulta: 8 de abril de 2013. URL: http:// www.educacioneningenieria.org/index.php/ edi/article/view/3/2

[16] A.J.D. Mosquera, M.J.C Mosquera y I. Artamónova. "Indicadores de capacidad aplicados a la deserción en las universidades colombianas". Ciencia e Ingeniería Neogranadina. Vol. $21 \mathrm{~N}^{\mathrm{o}} 2$ pp. 183-203. 2011. Fecha de consulta: 8 de abril de 2013. URL: http://www.redalyc.org/articulo. oa? id $=91123440010$

[17] L.K. Chan, S.W. Cheng and F.A. Spiring, "A new measure of Process Capability: Cpk". Journal of Quality Technology. Vol. $20 \mathrm{~N}^{\circ} 3$, pp. 162-175. 1988.

[18] W.L. Pearn, S. Kotz and N.L. Johnson. "Distributional and inferential properties of process capability indices". Journal of Quality Technology. Vol. 24, Issue 4, pp. 216-233. 1992.

[19] W. E. Carr. "A new process capability index: parts per million”. Quality Progress. Vol. 24, p. 152.1991.

[20] M. Greenwich and B.L. Jahr-Schaffrath. "A process incapability index". International 
Journal of Quality and Reliability Management. Vol. 12, Issue 4, pp. 58-71. 1994.

[21] P. A.Wright. "A process capability index sensitive to skewness". Journal of Statistical Computation \& Simulation. Vol. 52, pp. 195203. 1995.

[22] F.A. Spiring. "A Unifying Approach to Process Capability Indices". Journal of Quality Technology. Vol. 29, Issue 1, pp. 49-58. 1997.

[23] J.P. Chen and C.G. Ding. "A new process capability index for non-normal distributions". International Journal of Quality and Reliability
Management. Vol. 18, Issue 7, pp. 762-770. 2001.

[24] J.A. Clements. Process Capability Calculations for Non-Normal Distribution. Quality Progress. Vol. 24, Issue 9, pp. 95-100.1989.

[25] M. Perakis and E. Xekalaki. "A process capability index for discrete processes". Journal of Statistical Computation and Simulation. Vol. 75, Issue 3, p.185. 2005.

[26] Control Estadístico de la Calidad con MINITAB. Universidad Oberta de Catalunya. 2003. Fecha de consulta: 18 de abril de 2013. URL: http://www.uoc.edu/in3/e-math/docs/ SPC_6.pdf 